\title{
Percepções sobre a atuação multiprofissional frente a COVID-19: uma revisão integrativa de literatura com estudos geriátricos
}

\author{
Perceptions on multiprofessional performance in the face of COVID-19: an integrative literature \\ review with geriatric studies
}

Percepciones sobre el desempeño multiprofesional frente al COVID-19: una revisión de la

literatura integradora con los estudios geriátricos

Recebido: 28/07/2021 | Revisado: 03/08/2021 | Aceito: 04/08/2021 | Publicado: 09/08/2021

\author{
Gustavo Baroni Araujo \\ ORCID: https://orcid.org/0000-0002-3162-7477 \\ Universidade Estadual de Londrina, Brasil \\ E-mail: gustavobaroni13@hotmail.com \\ Thyago de Oliveira Afonso \\ ORCID: https://orcid.org/0000-0001-7616-9011 \\ Universidade Federal de Pernambuco, Brasil \\ E-mail: thyago.oafonso@gmail.com \\ Joelma Maria dos Santos da Silva Apolinário \\ ORCID: https://orcid.org/0000-0001-9521-9432 \\ Faculdade Maurício de Nassau, Brasil \\ E-mail: jo.silva00@hotmail.com \\ Mariana Pereira Barbosa Silva \\ ORCID: https://orcid.org/0000-0003-0852-8099 \\ Universidade Estadual do Piauí, Brasil \\ E-mail: marianapbsilvaa@gmail.com \\ Ranniely Kauany Bezerra da Silva \\ ORCID: https://orcid.org/0000-0002-7656-5185 \\ Universidade Federal de Pernambuco, Brasil \\ E-mail: rannykauany@hotmail.com \\ Pedro Adilon da Cruz Oliveira \\ ORCID: https://orcid.org/0000-0002-7425-4746 \\ Centro Universitário Mauricio de Nassau, Brasil \\ E-mail: pedroadilon123@gmail.com \\ Lorrane de Sousa Barbosa \\ ORCID: https://orcid.org/0000-0002-3668-2993 \\ Universidade Estadual da Paraíba, Brasil \\ E-mail: sousablorrane@gmail.com \\ Cézar Victor Alves de Lima \\ ORCID: https://orcid.org/0000-0002-9834-7066 \\ Universidade Estadual da Paraíba, Brasil \\ E-mail: cezarvictoralves@gmail.com
}

\begin{abstract}
Resumo
Durante a pandemia de COVID-19, os idosos (com idade cronológica de 65 anos e acima) são mais suscetíveis à infecção por SARS-CoV-2 devido à resposta alterada do sistema imunológico e à maior taxa de comorbidades subjacentes. A grande maioria das mortalidades é relatada em pacientes idosos. Assim, este estudo teve como objetivo avaliar alguns aspectos relacionados à reabilitação da COVID-19 em pacientes idosos e sua interação com multiprofissionais na área de saúde. Trata-se de uma revisão integrativa com o intuito de evidenciar a importância dos cuidados multiprofissionais aos idosos frente as condições da COVID-19. As bases de dados de literatura científica e técnicas consultadas foram: Literatura Latino-Americana e de Caribe em Ciências da Saúde (LILACS); Google Acadêmico, Scientific Electronic Library Online (SciELO). Os descritores foram selecionados a partir dos objetivos da pesquisa, sendo eles: "COVID-19" e "Idosos". Os critérios de inclusão foram artigos completos publicados na revista entre o período de janeiro de 2019 a maio de 2021 identificados em junho de 2021. No total, 9 artigos participaram deste trabalho. Dentre os principais resultados obtidos, destaca-se a chance de envolvimento de vários órgãos governamentais e diversas profissões. Os prejuízos da COVID-19 são mais comuns em pacientes idosos, entretanto, avaliações preventivas, multidisciplinares e holísticas são essenciais para reduzir as consequências da doença. Concluise neste trabalho, que a adoção dos cuidados multiprofissionais para a reabilitação da saúde, juntamente com a assistência técnica destes profissionais na prevenção, representa um grande impacto na melhoria da gestão do conhecimento, qualidade do atendimento e segurança do paciente idoso.
\end{abstract}

Palavras-chave: Saúde do idoso; COVID-19; Atuação multiprofissional. 


\begin{abstract}
During the COVID-19 pandemic, the elderly (chronological age 65 years and above) are more susceptible to SARSCoV-2 infection due to the altered immune system response and the higher rate of underlying comorbidities. The vast majority of mortalities are reported in elderly patients. Thus, this study aimed to evaluate some aspects related to the rehabilitation of COVID-19 in elderly patients and its interaction with multidisciplinary healthcare professionals. This is an integrative review with the aim of highlighting the importance of multidisciplinary care for the elderly in view of the conditions of COVID-19. The databases of scientific and technical literature consulted were: Latin American and Caribbean Literature on Health Sciences (LILACS); Google Scholar, Scientific Electronic Library Online (SciELO). The descriptors were selected based on the research objectives, namely: "COVID-19" and "Elderly". Inclusion criteria were full articles published in the journal between January 2019 and May 2021 identified in June 2021. In total, 9 articles participated in this work. Among the main results obtained, the chance of involvement of various government agencies and various professions stands out. COVID-19 damages are more common in elderly patients, however, preventive, multidisciplinary and holistic assessments are essential to reduce the consequences of the disease. It is concluded in this work that the adoption of multidisciplinary care for health rehabilitation, together with the technical assistance of these professionals in prevention, represents a great impact on improving knowledge management, quality of care and safety for elderly patients.
\end{abstract}

Keywords: Elderly health; COVID-19; Multidisciplinary action.

\title{
Resumen
}

Durante la pandemia de COVID-19, los ancianos (edad cronológica de 65 años o más) son más susceptibles a la infección por SARS-CoV-2 debido a la respuesta alterada del sistema inmunológico y la mayor tasa de comorbilidades subyacentes. La gran mayoría de las muertes se notifican en pacientes ancianos. Así, este estudio tuvo como objetivo evaluar algunos aspectos relacionados con la rehabilitación del COVID-19 en pacientes ancianos y su interacción con profesionales sanitarios multidisciplinares. Se trata de una revisión integradora con el objetivo de resaltar la importancia de la atención multidisciplinar al anciano ante las condiciones del COVID-19. Las bases de datos de literatura científica y técnica consultadas fueron: Literatura Latinoamericana y del Caribe en Ciencias de la Salud (LILACS); Google Scholar, Scientific Electronic Library Online (SciELO). Los descriptores fueron seleccionados en función de los objetivos de la investigación, a saber: "COVID-19" y “Anciano". Los criterios de inclusión fueron artículos completos publicados en la revista entre enero de 2019 y mayo de 2021 identificados en junio de 2021. En total, participaron 9 artículos en este trabajo. Entre los principales resultados obtenidos, destaca la posibilidad de participación de diversos organismos gubernamentales y diversas profesiones. Los daños del COVID-19 son más comunes en pacientes ancianos, sin embargo, las evaluaciones preventivas, multidisciplinarias y holísticas son fundamentales para reducir las consecuencias de la enfermedad. Se concluye en este trabajo que la adopción de cuidados multidisciplinares para la rehabilitación de la salud, junto con la asistencia técnica de estos profesionales en prevención, representa un gran impacto en la mejora de la gestión del conocimiento, la calidad de la atención y la seguridad del paciente anciano.

Palabras clave: Salud de los ancianos; COVID-19; Acción multidisciplinar.

\section{Introdução}

A pandemia 2 (SARS CoV-2), também conhecida como doença coronavírus 2019 (COVID-19), nos coloca na vanguarda dos cenários com graves consequências para os sistemas de saúde no Brasil e no mundo. A pneumonia intersticial viral atual provoca grave insuficiência respiratória hipoxêmica, que tem resultado em superlotação em unidades de terapia intensiva (UTI), escassez de equipamento e pessoal e mortalidade significativa, especialmente em populações em risco que têm condições de saúde debilitadas.

Cada profissional treinado torna-se um agente multiplicador desse procedimento, contribuindo interna e externamente à área hospitalar para reduzir as taxas de infecção de COVID 19 já que o procedimento de vestir e despir vai além de uma simples colocação e retirada de roupas, incluindo ação preventiva é essencial realizada antes e após qualquer procedimento (Barbosa, 2020).

Fora e no contexto do COVID 19, há boas evidências de que os idosos com fragilidade e multimorbidade que sobrevivem a doenças graves e intensos cuidados são mais propensos do que grupos mais jovens e mais saudáveis a experimentar complicações, incluindo descondicionamento físico grave, falência de múltiplos órgãos, disfunção pulmonar, cognitiva, transtorno de estresse pós-traumático e pós-intensivo síndrome do cuidado (Valença et al., 2017); (Romero et al., 2021).

A reabilitação geriátrica usa abordagens baseadas em evidências para apoiar a reabilitação de pessoas idosas com fragilidade e multimorbidade após uma doença aguda. O objetivo é permitir que os pacientes se recuperem e participem tanto da 
vida social quanto possível. Os princípios fundamentais da reabilitação geriátrica foram definidos recentemente (The Lancet, 2020).

Diante deste contexto, o objetivo deste estudo é realizar um estudo de cunho de revisão integrativa com características exploratórias sobre o tema de reabilitação geriátrica e percepções sobre a COVID - 19 de modo global. Para que os objetivos deste estudo fossem alcançados, foram utilizados como ferramentas bibliográficas artigos publicados referentes ao cuidado multiprofissional e a COVID-19.

\section{Metodologia}

Trata-se de uma revisão integrativa com o intuito de evidenciar a importância dos cuidados multiprofissionais aos idosos frente as condições da COVID-19 partir de um conjunto de estudos que abordam essa temática. Optou-se por este método entendendo que se trata de uma estratégia para identificar e analisar as evidências existentes de práticas de saúde no processo de recuperação dessa população.

A revisão integrativa é uma metodologia de investigação que permite a procura, a avaliação crítica e a síntese de evidências existentes na literatura científica sobre determinado tema investigado, em que o produto final é o estado do conhecimento, a elaboração e a implementação de intervenções efetivas na prestação de cuidados e na redução de custos. Além disso, permite a identificação de fragilidades, que poderão conduzir ao desenvolvimento de futuras investigações (Sousa, Marques-Vieira, Severino, \& Antunes, 2017)

Para a confecção dessa revisão, utilizou-se as seguintes etapas: 1) Seleção das questões temáticas; 2) Estabelecimento dos critérios para a seleção da amostra, 3) Representação das características da pesquisa original; 3) Interpretação dos resultados; e 4) Apresentação da revisão (Ganong 1987).

As bases de dados de literatura científica e técnicas consultadas foram: Literatura Latino-Americana e de Caribe em Ciências da Saúde (LILACS); Google Acadêmico, Scientific Electronic Library Online (SciELO). Os descritores foram selecionados a partir dos objetivos da pesquisa, sendo eles: "COVID-19" e "Idosos". Os critérios de inclusão foram artigos completos publicados na revista entre o período de janeiro de 2019 a maio de 2021 identificados em junho de 2021. Somente foram incluídos artigos que tivessem alguma relação com a saúde do idoso e COVID-19 relacionado ao cuidado multidisciplinar. Foram excluídos resumos (simples e expandidos) apresentados em eventos científicos e resumos (simples ou expandidos) publicados em anais de eventos científicos.

Posteriormente a aplicação dos critérios de inclusão e exclusão, os trabalhos foram selecionados e avaliados por título e resumo com o propósito de direcionar a temática para este estudo. Esta avaliação foi realizada por um pesquisador. Para os casos em que a leitura do resumo não fosse suficiente para definir a inclusão do artigo, foram considerados os demais critérios e a leitura na íntegra. Os dados de interesse dos trabalhos selecionados foram extraídos e registrados em planilha padronizada, que continha as principais informações sobre cada artigo.

A elegibilidade de cada estudo foi determinada pela leitura na íntegra e sua identificação é demonstrada no fluxograma da Figura 1. O fluxograma embasado no Preferred reporting items for systematic reviews and meta-analyses (PRISMA) sintetiza a busca dos artigos que compuseram a amostra final da revisão. 
Figura 1: Fluxograma do processo de identificação e seleção de artigos.
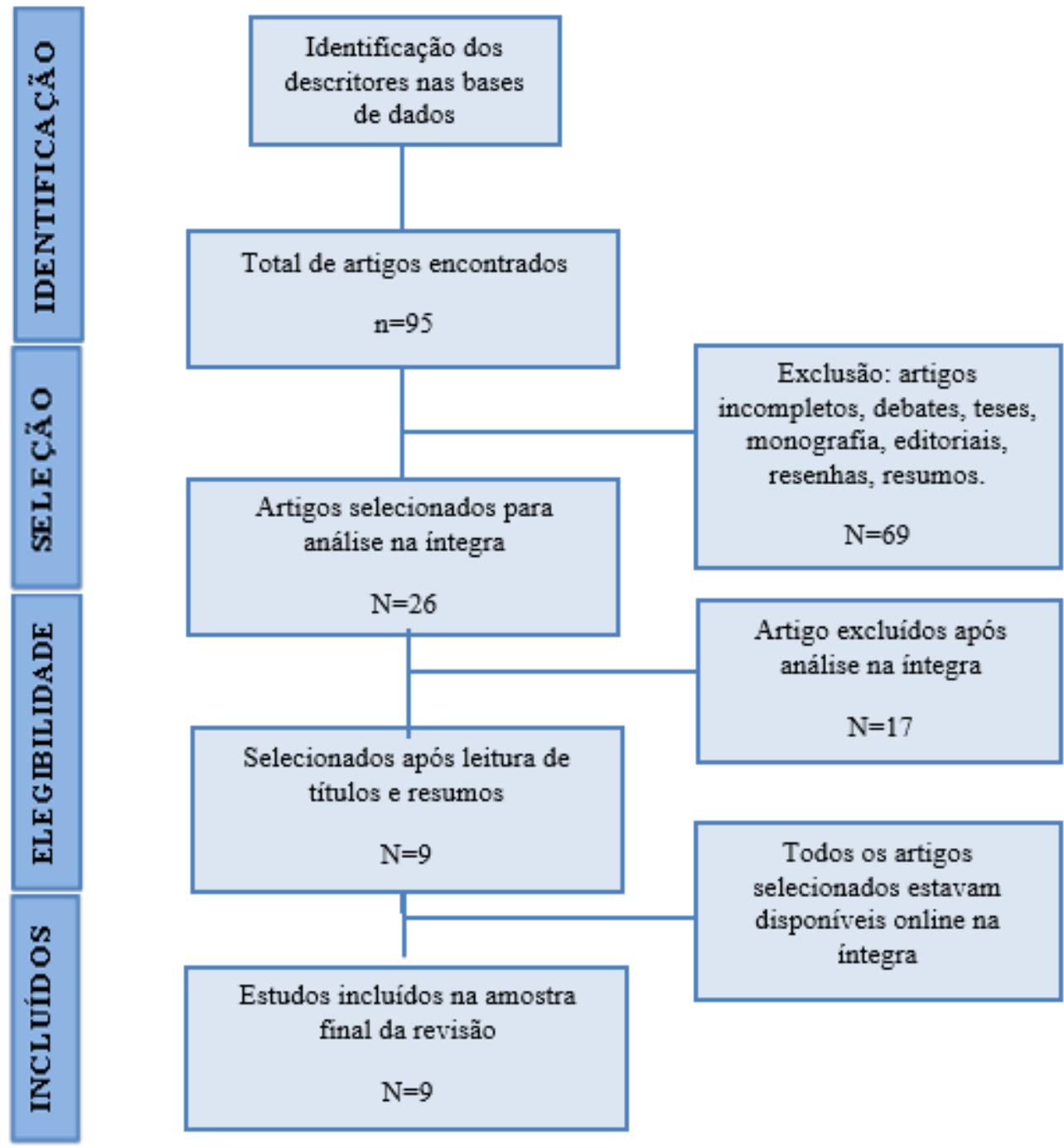

Fonte: Elaborado pelo autor a partir do Fluxograma PRISMA (2021).

Na figura 1 observa-se que a partir da coleta de dados, localizaram-se 95 estudos, dos quais 69 foram excluídos por não atenderem aos critérios de inclusão, restando 26 artigos, que após leitura dos títulos e resumos obteve-se uma amostra de 26 estudos, na primeira etapa da avaliação.

Na segunda etapa, procedeu-se a leitura dos 26 estudos, desses, 17 foram excluídos por não responder à questão norteadora. Ao final foram incluídos 9 estudos que se adequaram ao objetivo proposto pela pesquisa. Diante disso, os estudos incluídos para análise final foram organizados em uma planilha de dados contendo as seguintes variáveis: autor, título, ano e metodologia empregada (Quadro 1) 
Quadro 1: Caracterização dos estudos incluídos na revisão segundo autores, título ano de publicação e metodologia.

\begin{tabular}{|c|c|c|c|c|}
\hline $\mathbf{N}^{\mathbf{0}}$ & Autor & Título & Ano & Metodologia \\
\hline 1 & Machado et al. & $\begin{array}{l}\text { Estimativas de impacto da } \\
\text { COVID-19 na mortalidade } \\
\text { de idosos } \\
\text { institucionalizados no } \\
\text { Brasil }\end{array}$ & 2020 & Estudo transversal e de simulação \\
\hline 2 & Moraes et al. & $\begin{array}{l}\text { Violência contra idosos } \\
\text { durante a pandemia de } \\
\text { Covid-19 no Brasil: } \\
\text { contribuições para seu } \\
\text { enfrentamento }\end{array}$ & 2020 & Estudo de revisão \\
\hline 3 & Moraes et al. & $\begin{array}{l}\text { COVID-19 nas instituições } \\
\text { de longa permanência para } \\
\text { idosos: estratégias de } \\
\text { rastreamento laboratorial e } \\
\text { prevenção da propagação } \\
\text { da doença }\end{array}$ & 2020 & Estudo descritivo exploratório \\
\hline 4 & Souza & $\begin{array}{l}\text { Isolamento social versus } \\
\text { qualidade de vida dos } \\
\text { idosos: um } \\
\text { olhar multiprofissional } \\
\text { frente à pandemia do } \\
\text { Covid-19 }\end{array}$ & 2020 & Estudo descritivo exploratório \\
\hline 5 & Peixoto et al. & $\begin{array}{l}\text { Barreiras e facilitadores do } \\
\text { trabalho multiprofissional } \\
\text { em saúde na pandemia da } \\
\text { COVID-19 }\end{array}$ & 2020 & Estudo descritivo transversal \\
\hline 6 & Theodosio et al. & $\begin{array}{l}\text { Barreiras e facilitadores do } \\
\text { trabalho multiprofissional } \\
\text { em saúde na pandemia da } \\
\text { COVID-19 }\end{array}$ & 2021 & Estudo descritivo exploratório \\
\hline 7 & Unicovsky; Santarem & $\begin{array}{l}\text { Desafios para Enfermagem } \\
\text { no atendimento a os } \\
\text { pacientes idosos com } \\
\text { delirium e COVID-19 em } \\
\text { unidades de cuidados } \\
\text { críticos }\end{array}$ & 2021 & Estudo descritivo e reflexivo \\
\hline 8 & $\begin{array}{l}\text { Matioli; } \quad \text { Benati; } \\
\text { Santos }\end{array}$ & $\begin{array}{l}\text { Atendimento domiciliar } \\
\text { odontológico ao idoso em } \\
\text { tempos de pandemia por } \\
\text { COVID-19 }\end{array}$ & 2021 & Estudo de revisão bibliográfica \\
\hline 9 & Santos et al. & $\begin{array}{l}\text { Adaptações nos serviços de } \\
\text { atenção primaria à saúde } \\
\text { frente ao COVID-19: } \\
\text { Vivencias } \\
\text { multiprofissionais }\end{array}$ & 2021 & Estudo qualitativo descritivo \\
\hline
\end{tabular}

Fonte: Autores (2021).

\section{Resultados e discussão}

O objetivo deste estudo foi realizar um estudo de cunho de revisão integrativa com características exploratórias sobre o tema de reabilitação geriátrica e percepções sobre a COVID - 19 de modo global.

No que se refere as metodologias, os resultados observados apresentam a maior prevalência encontrada em estudos de caráter descritivo $(66,6 \%)$ esse dado pode ser parcialmente explicado considerando a relação entre o impacto da pandemia de 
COVID-19 sobre a população idosa e esta abordagem metodológica que tem como objetivo descrever as características de uma população, um fenômeno ou experiência para o estudo realizado. É importante ressaltar que essa metodologia é realizada baseando-se em aspectos na formulação das perguntas que norteiam a pesquisa, estabelecendo uma relação entre as variáveis propostas no objeto de estudo em análise (Lakatos \& Marconi, 2001).

Dois artigos $(22,2 \%)$ tratam de revisão de literatura. Essa metodologia adotada parece positiva no sentido de conseguir avançar em determinado campo do conhecimento, partindo do princípio de que é necessário primeiro conhecer o que já se tem na literatura cientifica a partir de trabalhos realizados por outros pesquisadores. Considerando que a COVID-19 é um tema recente na literatura cientifica, essa abordagem pode fornecer sustentação teórica para a disseminação do conhecimento sobre a temática investigada, bem como fomentar o desenvolvimento de novas pesquisas (Caldas, 1986).

Os resultados dos trabalhos investigados tratam o processo de reabilitação de idosos com Covid-19 sendo realizado por uma equipe multiprofissional composta por enfermeiras, terapeutas, médicos, assistentes sociais, psicólogos e outros grupos profissionais (por exemplo, farmacêuticos) que estruturam a reabilitação em torno do necessidades e objetivos individuais dos pacientes e suas famílias de acordo com os princípios da geriatria abrangente avaliação.

Dada a extensão em que os idosos foram afetados por COVID-19 durante a pandemia, e dada a emergência, evidências de deficiência complexa de longo prazo como consequência de infecção por SARS-CoV-2, é provável ser um crescimento na demanda por reabilitação geriátrica em nos próximos meses e anos. Isso acontece enquanto a capacidade e os recursos em reabilitação geriátrica estão diminuindo por causa da pandemia (The Lancet, 2020).

Internacionalmente, tem-se visto membros de equipes multidisciplinares de reabilitação elaborados em hospitais para apoiar os cuidados agudos de COVID, enquanto as instalações de reabilitação foram rebatizadas de leitos médicos subagudos ou foram usadas para fornecer camas de rebaixamento seguras para COVID para proteger instalações de cuidados de longa duração e lares de idosos de casos COVID-positivos (Gomes, 2021).

Tentativas de 'conectar o gap ', fornecendo um recurso financeiro adicional para apoiar cuidados de base comunitária nem sempre foram bem considerados, uma vez que carecem da reabilitação necessária infraestrutura para permitir a reabilitação geriátrica baseada em evidências. Prevenção de infecção e medidas de controle têm mais recursos esgotados porque o distanciamento social e corte requisitos significam que a capacidade das instalações que permanecer é reduzida (dados próprios, pesquisa com oito países europeus) (Silva 2019).

Isso representa um paradoxo da reabilitação. Necessita-se de reabilitação geriátrica agora mais do que nunca, mas a própria pandemia que gerou uma demanda sem precedentes também esgotou os recursos necessários para atender às necessidades de nossos pacientes (Silva, 2021); (Duarte et al., 2021). O impacto da pandemia em outros aspectos da saúde, incluindo cuidados com o câncer e cuidados com o AVC, foi bem documentado e tem sido o foco de muita advocacia nacional e internacional.

Não foi visto um esforço semelhante em torno da reabilitação geriátrica e ainda é provável, conforme essas condições, que a falha em entregar até mesmo níveis de rotina de reabilitação para pacientes mais velhos terão resultado em uma demanda reprimida - uma epidemia de fragilidade e deficiência ainda não totalmente quantificada. Planos emergentes para reabilitação em face da COVID-19 que dependem fortemente da experiência de reabilitação para a síndrome de fadiga crônica, ou que usam informações soluções baseadas em tecnologia são improváveis de abordar totalmente as necessidades dos idosos com fragilidade e multimorbidade (Silva, 2019); (Hammerschmidt; Santana, 2020).

Muitos idosos vivem sozinhos e são incapazes de se envolver com tais tecnologia devido a deficiência cognitiva, sensorial ou física A curto prazo, devemos defender urgentemente o caso de proteger os recursos de reabilitação geriátrica de mais desnudação. Eles constituem uma parte importante do COVID-19 resposta. Tirando recursos deles para apoiar o mesmo, a resposta é contra-intuitiva. À medida que avançamos para uma manutenção e fase de recuperação da pandemia, devemos garantir que a liberação de recursos para a reabilitação geriátrica é priorizada. 
Compreender o tamanho do desafio e o que as intervenções funcionam melhor no contexto do COVID-19 recuperação também é essencial. No longo prazo, devemos enfrentar o fato de que a reabilitação geriátrica tem, até agora, recebido prioridade inadequada em saúde sistemas internacionais (The Lancet, 2020).

Isso se manifestou de maneira diferente entre países. Na Alemanha, seu desenvolvimento foi realizado por sucessivos períodos de reabilitação e sistemas de pagamento parcialmente fixos em reabilitação pós-aguda de pacientes internados, que têm flexibilidade insuficiente para acomodar as necessidades complexas dos mais deficientes.

As capacidades dos serviços de reabilitação ainda são insuficientes. No Brasil, os geriatras encontraram seu treinamento e implantação cada vez mais focados na prestação de cuidados agudos às despesas de reabilitação, embora limites de tempo arbitrários na reabilitação baseada na comunidade serviram, mais uma vez, para desinvestir os mais deficientes. Na Holanda, a eficiência da economia deixou o setor, antes foco de admiração internacional, com flexibilidade limitada para responder às circunstancias causadas pela pandemia.

Agora, quando enfrenta-se uma pandemia de necessidade de reabilitação não atendida em idosos com fragilidade e multimorbidade, a reabilitação geriátrica poderia desempenhar um papel transformador semelhante se protegermos e desenvolvermos nossos serviços para atender à necessidade (Souza, 2021).

A relevância do trabalho parte da necessidade das discussões a partir de informações já disponíveis na literatura a respeito da atuação multiprofissional frente as condições da COVID-19 em idosos, reforçando a importância das diversas contribuições cientificas para que exista a qualificação de recursos humanos para atuar com essa população através da atualização de conhecimento em saúde. Estudos futuros poderiam analisar, de forma prática, as percepções destes profissionais, bem como abordar as questões práticas enfrentadas durante a pandemia de COVID-19 verificando em que medida estas estão articuladas com os aspectos teóricos para melhor compreensão sobre a temática investigada.

\section{Considerações Finais}

É possível concluir que as capacitações realizadas possibilitaram a troca de experiências, reflexões sobre uma cultura de segurança e aprendizagem bidirecional entre residentes e saúde profissionais, e também lidar com o novo, a indecisão e o desconhecido foi um grande desafio.

A educação em saúde está presente cotidianamente nas relações interprofissionais, pois se baseia em uma prática em que o conhecimento técnico deve proporcionar o empoderamento da saúde profissional nessa troca de experiências. Além disso, promove-se a autonomia de residentes como promotores de conhecimentos em seu contexto de saúde geral na pandemia.

Neste momento, a adoção de reabilitações para a saúde e assistência técnica profissionais na prevenção, representa um grande impacto na melhoria da gestão do conhecimento, qualidade do atendimento e segurança do paciente.

A relevância do trabalho parte da necessidade das discussões a partir de conteúdos existentes na literatura a respeito da atuação multiprofissional frente as condições da COVID-19 em idosos, reforçando a importância das diversas contribuições científicas para que exista a qualificação de recursos humanos para atuar com essa população através da atualização de conhecimento em saúde. Estudos futuros poderiam analisar, de forma prática, as percepções destes profissionais, bem como abordar as questões práticas enfrentadas durante a pandemia de COVID-19 verificando em que medida estas estão articuladas com os aspectos teóricos para melhor compreensão sobre a temática investigada.

\section{Referências}

Brasil. (2020). Ministério da Saúde. Secretaria de Vigilância em Saúde. Orientação para Manejo de Pacientes com COVID-19.

Barbosa, J. A. G. (2020). Estado nutricional e o enfrentamento da COVID-19: reflexões para a prática de Enfermagem. Braz. J. Hea. Rev., Curitiba, 3 (3), 66716675 . 
Barbosa, I. R. et al. (2020). Incidência e mortalidade por COVID-19 na população idosa brasileira e sua relação com indicadores contextuais: um estudo ecológico. Rev. Bras. Geriatr. Gerontol. Alagoas, v.23, n.1.

Caldas, M. A. E. (1986). Estudos de revisão de literatura: fundamentação e estratégia metodológica. Hucitec,

Duarte Ferreira Neto, P., Wilma Fernandes Rosendo, C., Anselmo Silva de Lima, F., Patrícia Ferreira Bezerra, Y., Medeiros de Araújo Nunes, V., \& Pereira da Silva Lima, S. (2021). O impacto da COVID-19 na saúde de pessoas institucionalizadas. Revista Ciência Plural, 7(2), 196-210.

Fuganti, C. L.et al. (2020). Parecer BRASPEN/ AMIB para o Enfrentamento do COVID-19 em Pacientes Hospitalizados. Braspen Journal , 3 - 5.

Ganong, L. H. (1987). Integrative reviews of nursing research. Research in Nursing \& Health, New York, 10 (11), 1-11.

Gomes, M. A. C., Fernandes C. S., Fontenele N. A. O., Galindo Neto N. M., Barros L. M., Frota N. M. (2021). Elderly people's experience facing social isolation in the COVID-19 pandemic. Rev Rene.;22:e69236

Hammerschmidt K. S. A., \& Santana R..F. (2020). Saúde do idoso em tempos de pandemia Covid-19. Cogitare enferm.

Lakatos, E. M., \& Marconi, M. A. (2001). Fundamentos metodologia científica. (4.ed.), Atlas.

Nakamura, L. et al. (2020). Correlação entre produtividade, depressão, ansiedade, estresse e qualidade de vida em residentes multiprofissionais em saúde. Brazilian Journal of Development, 6(12), 96892-905.

Romero, D. E. et al. (2021). Idosos no contexto da pandemia da COVID-19 no Brasil: efeitos nas condições de saúde, renda e trabalho. Cadernos de Saúde Pública [online].

Schmidt, B .et al. (2020). Saúde mental e atenção psicossocial na pandemia COVID19: orientações às/aos psicólogas/os hospitalares. Rio de Janeiro: Fiocruz/CEPEDES, 22 p. Cartilha.

Silva, A. F. et al. (2019). Presenteeism in multi professional team workers in the Adult Intensive Care Unit. Revista Brasileira de Enfermagem, 72, 96-104.

Silva, J. C. et al. (2015). Percepção dos residentes sobre sua atuação no programa de residência multiprofissional. Acta Paulista de Enfermagem, 28(2), 132- 8.

Silva, L. B. Residência Multiprofissional em Saúde no Brasil: Alguns aspectos da trajetória histórica. Revista Katálysis, 21 (1), 200-209, 2018.

Silva, T. S. da., Nascimento, L. S. do., Rabelo, A. R. de M., Brito, J. S. de., Rosas, M. A ., Cavalcanti, G. L. O. S. ., \& Marcelino, J. F. de Q. . (2021). Qualidade de vida dos residentes de um Programa Multiprofissional Integrado em Saúde na pandemia da COVID-19. Research, Society and Development, 10(5), e35110513637. https://doi.org/10.33448/rsd-v10i5.13637.

Sousa, L. M. M., Marques-Vieira, C. M. A., Severino, S. S. P., \& Antunes, A. V. (2017). A metodologia de revisão integrativa da literatura em enfermagem. Revista Investigação em Enfermagem, 21(2), 17-26.

Souza, C. S. et al. Cultura de segurança em unidades de terapia intensiva: perspectiva dos profissionais de saúde. Revista Gaúcha de Enfermagem, v. 40, p. e20180294, 2019.

Souza, W. S. de, Comassetto, I., Junqueira, T. L. S. ., Souza, E. M. S. de, Oliveira, A. dos S., \& Leão, A. L. (2021). Vivência da Equipe Multiprofissional de Saúde no enfrentamento da COVID-19 em Serviços de Internação Hospitalar. Research, Society and Development, 10(4), e25910414048. https://doi.org/10.33448/rsd-v10i4.14048.

The Lancet. (2020). COVID-19: protecting health-care workers. The Lancet, 395(10228), 922.

Valença. T. D.C. et al. (2017). Deficiência física na velhice: Um estudo estrutural das representações sociais. 